\title{
XLI. The Sun's motion with respect to the Æther
}

\section{C.V. Burton D.Sc.}

To cite this article: C.V. Burton D.Sc. (1910) XLI. The Sun's motion with respect to the $/$ ther , Philosophical Magazine Series 6, 19:111, 417-423, DOI: 10.1080/14786440308636815

To link to this article: http://dx.doi.org/10.1080/14786440308636815

曲 Published online: 21 Apr 2009.

Submit your article to this journal

Џ Article views: 3

Q View related articles $₫$ 
XLI. The Sun's Motion with respect to the Ether. $B y$ C. V. Burtor, D.Sc.*

1. QOME time ago, in a letter to 'Nature,' I ventured to

point out that the Sun's velocity with respect to the rether could be deduced from the observed times of the eclipses of Jupiter's satellites. At that time it was unknown to me that the following remark on the same subject had been made by Maxwellt:-

"The only practicable method of determining directly the relative velocity of the rether with respect to the solar system is to compare the values of the velocity of light deduced from the observation of the eclipses of Jupiter's satellites when Jupiter is seen from the earth at nearly opposite points of the ecliptic."

If for brevity we speak of the motion of the ather with respect to the solar system as a wind, the essence of the matter might be expressed by saying that light from Jupiter would reach us more quickly when that planet was to windward of us than when it was to leeward; the wind-velocity being in the former case added to, and in the latter case subtracted from, the velocity of propagation. Maxwell's reference to Jupiter as "seen from the earth at nearly" opposite points of the ecliptic" is evidently a mere slip. The context makes it clear that the very simple essentials of the problem had been fully realized.

2. Though some thirty years have elapsed since Maxwell wrote, attention does not seem to have been directed to the subject in any effective degree; yet from the standpoint of electromagnetic theory, great interest attaches to any physically possible method of determining motion with respect to the xther; while from an astronomical point of view the results to be looked for are no less important. Unfortunately, owing to the small inclination of Jupiter's orbit to the ecliptic, we are virtually restricted to a problem in two dimensions. The present would seem to be a favourable time for directing the attention of astronomers and physicists to these questions anew; on the one hand because Prof. Kapteyn's discovery of two principal star-drifts makes it important to determine the motion of each drift with respect to the xther, so far as we can ; and on the other hand because the material needed for the computations will soon be available. The Harvard photometric observations of the eclipses have been very fully discassed by Prof. R. A. Sampson, in

* Communicated by the Physical Society : read Norember 26, 1909.

† Encyclopeedia Britannica, 9th ed., article "Ether."

Phil. Mag. S. 6. Vol. 19. No. 111. March 1910. $2 \mathrm{E}$ 
a memoir which is about to appear as vol. lii. pt. ii. of the Harvard Annals*.

3 . Let the origin of coordinates coincicle always with the sun's centre, the coordinate axes being in directions fixed in space. At any time $t$ let the coordinates of Jupiter's satellite I. be $\left(x^{\prime}, y^{\prime}, z^{\prime}\right)$ and those of the earth $(. x, y, z)$; let the velocity of the sun with respect to the æther have components $(a, b, c)$ in the directions of the coordinate axes, the assumption being made that this velocity does not vary sensibly within the period covered by the observations considered.

4. Let an eclipse of satellite I. be observed at time $t^{\prime}$; then the eclipse actually occurred at some previous time $t=t^{\prime}-\tau$, when the coordinates of the satellite were

$$
\left\{x^{\prime}-\left(a+\dot{x}^{\prime}\right) \tau, \quad y^{\prime}-\left(b+\dot{y}^{\prime}\right) \tau, \quad z^{\prime}-\left(c+\dot{z}^{\prime}\right) \tau\right\} ; .
$$

the axes of reference being here understood to have the same position in the cether as our heliocentric axes have at time $t$. In the interval of time $\tau$, therefore, light has travelled through the ether from (1) to a point whose coordinates, referred to the same axes, are $(x, y, z)$. It may be provisionally assumed that $a+\dot{x}^{\prime}, b+\dot{y}^{\prime}, c+\dot{z}^{\prime}$ are small compared with $\mathrm{V}$, the velocity of light; the distance between (1) and $(x, y, z)$ is then approximately

$$
r+\frac{\tau}{r}\left\{\xi\left(a+\dot{x}^{\prime}\right)+\eta\left(b+\dot{y}^{\prime}\right)+\zeta\left(c+\dot{z}^{\prime}\right)\right\} ; .
$$

where

$$
\xi_{;}^{*} \eta, \zeta \equiv x-x^{\prime}, y-y^{\prime}, z-z^{\prime} \text {, and } r^{2} \equiv\left(x-x^{\prime}\right)^{2}+\left(y-y^{\prime}\right)^{2}+\left(z-z^{\prime}\right)^{2}
$$

5. Since (2) has to be equated to $\mathrm{V} \tau$, each observation furnishes an equation of the form

$$
\mathrm{V} \tau-r-\tau / r \cdot\left\{\xi\left(a+x^{\prime}\right)+\eta\left(b+\dot{y}^{\prime}\right)+\zeta\left(c+\dot{i}^{\prime}\right)\right\}=0 ;
$$

or, remembering that $r / \tau$ is very approximately equal to $\mathrm{V}$,

$$
\xi\left(a+\dot{x}^{\prime}\right)+\eta\left(b+\dot{y}^{\prime}\right)+\zeta\left(c+\dot{z}^{\prime}\right)-\mathrm{V}\left(\mathrm{V} \tau-r^{\prime}\right)=0 .
$$

Now $\left(\dot{x}^{\prime}, \dot{y}^{\prime}, \dot{z}^{\prime}\right)$ is the resultant of Jupiter's orbital velocity and of the velocity of satellite 1 . in its orbit around the planet. At the time of an eclipse it may amount, perhaps, to 22 kilom. per second, which is about half the smallest possible estimate of the probable error in the most favourably conditioned velocity-component $(\alpha)$ [see (11) below]. Moreover, the error introduced into $a, b$, or $c$ by neglecting

* Since this note was written Prof. Sampson has faroured me with some advance sheets of his work, wherein are tabulated just those residual errors from which the Sun's velocity has to be computed. 
$\ddot{v}^{\prime}, \dot{y}^{\prime}$, and $\dot{z}^{\prime}$ would be a very small fraction of 22 kilom. per second, and accordingly equations (4) may be simplified by the omission of $\dot{x}^{\prime}, \dot{y}^{\prime}, \dot{z}^{\prime}$.

6. These simplified equations can be treated by the method of least squares. In this preliminary discussion, all the observations are taken to be of equal weight, so that we have simply to make the sum of the squares of the left-hand members of these equations a minimum with respect to $a, b$, and $c$. Thus

$$
\left.\begin{array}{l}
a \Sigma \xi^{2}+b \Sigma \xi \eta+c \Sigma \zeta \xi=\mathrm{V} \Sigma \xi\left(\mathrm{V}_{\tau}-r\right) \\
a \Sigma \xi \eta+b \Sigma \eta^{2}+c \Sigma \eta \zeta=\mathrm{V} \Sigma \eta\left(\mathrm{V}_{\tau}-r\right) \\
a \Sigma \zeta \xi+b \Sigma \eta \zeta+c \Sigma \zeta^{2}=\mathrm{V} \Sigma \zeta\left(\mathrm{V}_{\tau}-r\right)
\end{array}\right\} ; .
$$

and from these equations values for $a, b, c$ can be at once written down.

7. Before discussing the choice of axes, it will be convenient to obtain general expressions for the probable errors of $a, b$, and $c$. The residuals $\mathrm{V} \tau-r$ may be regarded as the sole source of error, since all the other quantities involved in (5) are known with ample accuracy for our purpose. In dealing with the various errors which may affect the values adopted for the quantities $\mathrm{V} \tau-r$, let the suffix o distinguish true values, while the suffix $s$ indicates those quantities which are variable from one $\mathrm{V} \tau-r$ to another. Thus let

$$
\mathrm{V}=\mathrm{V}_{0}+v, \quad r=r_{0}+k r_{0} ; \quad \cdot \quad . \quad .
$$

so that $v$ is the error in the accepted value $V$ for the velocity of light, and $k$ is the proportional error in the value assigned to the solar parallax. (The angular magnitudes involved in our estimate of $r$ can be so accurately ascertained that no appreciable error can arise from them.)

8. Again, let $\left(\tau_{0}\right)_{s}$ denote the time actually taken by light to travel from the satellite at mid-eclipse to the observer. Then, $\left(\tau_{0}\right)_{s}$ being, the difference between the apparent and the actual time of mid-eclipse, the value $\tau_{s}$ which is a vailable to us will be liable to differ from $\left(\tau_{0}\right)_{s}$ owing to three sources of error:- -

(i.) The irregularity of Jupiter's surface causes the actual eclipses to succeed one another at intervals of time which are sensibly discrepant from those calculated theoretically. Thus $\tau_{s}$ is affected by an error of prediction which is periodic or quasi-periodic * in character, and which we denote by $\chi_{\text {. }}$.

* Though Prof. Sampson tells me that no period has been recounized in the residual errors. 
(ii.) There will also necessarily be some slight uncertainty as to the epoch of the actual eclipses, and $\tau_{s}$ will on this account be liable to a small constant error $(\gamma)$.

(iii.) In determining the apparent time of mid-eclipse, an error of observation is incurred; let the error thus introduced into the value of $\tau_{s}$ be called $v_{s}$; we have accordingly

$$
\boldsymbol{\tau}_{s}=\left(\tau_{0}\right)_{s}+\chi_{s}+\gamma+v_{s} \text {. . . . . . }
$$

9. For any single eclipse-observation, to a sufficient approximation,

$$
\mathrm{V}_{\tau}-r=\mathrm{V}_{0} \tau_{0}-r_{0}+\mathrm{V}\left(\boldsymbol{v}_{\mathrm{s}}+\chi_{s}+\gamma\right)+\tau v-k r . .
$$

Without, in the first place, taking account of the motion of the solar system through the ather, we may make $\gamma$ very small by making use of a number of eclipse-observations. extending over at least one Jovian year. Moreover, $\gamma$ only enters into our results in association with factors which tend approximately to disappear in the long run; its effect may therefore be disregarded. For similar reasons our final results will not be appreciably affected by the small errors 2 and $l$. (When the numerical values of $a, b, c$ have been found from (5), it will be possible, without much additional labour, to determine more definitely the influence of given errors $\gamma, v, k$ on the values found for $a, b, c$, as well as the probable errors in $a, b, c$ arising from an assumed "all round" value for the probable observational error corresponding to a single eclipse. For the present we must be content with a more tentative estimate of the accuracy to be expected.)

10. Finally, since the residual effects for which we are searching depend on the direction of the line drawn from the earth to Jupiter, and have thus a principal period of about 12 years, it is especially important for our purpose that the Jovian system should be free from any influence having an approximately equal period, and capable of affect-. ing the times of eclipse of the first satellite. Prof. Sampson lindly went into this question with me, and found that no disturbing influence of the period in question was to be feared. We may accordingly confine our attention (in this preliminary discussion at least) to the errors typified by $\chi_{s}+v_{s}$.

11. Referring now to equations (5), we find that the error $\chi_{s}+v_{s}$ in a single observation gives rise to an error in $a$ equal to

$$
\frac{\mathrm{V}^{2}\left(\chi_{s}+v_{s}\right)}{\mathrm{D}}\left|\begin{array}{lll}
\xi_{s} & \Sigma \xi_{\eta} & \Sigma \zeta \xi \\
\eta_{s} & \Sigma \eta^{2} & \Sigma \eta \zeta^{\prime} \\
\zeta_{s} & \Sigma \eta \zeta & \Sigma \zeta^{2}
\end{array}\right| ; \text { where } \mathrm{D} \equiv\left|\begin{array}{ccc}
\Sigma \xi^{2} & \Sigma \xi \eta & \Sigma \zeta \xi \\
\Sigma \xi_{\eta} & \Sigma \eta^{2} & \Sigma \eta \zeta \\
\Sigma \zeta \xi & \Sigma \eta \zeta & \Sigma \zeta^{2}
\end{array}\right|
$$


Thus if $\epsilon$ represents the all-round probable value of the error $\chi_{s}+v_{s}$ for a single eclipse, we shall have

$$
\begin{aligned}
& \text { p.e. in } a=\frac{\mathrm{V}^{2} \epsilon}{\mathrm{D}}\left\{\Sigma\left|\begin{array}{ccc}
\xi_{s} & \Sigma \xi \eta & \Sigma \zeta \xi \\
\vdots & \Sigma \eta^{2} & \Sigma \eta \zeta \\
\eta_{s} & \Sigma \zeta_{s}^{2} \\
\zeta_{s} & \Sigma \eta \zeta & \Sigma \zeta^{2}
\end{array}\right|^{2}\right\}^{\frac{1}{2}} \\
& =\mathrm{V}^{2} \epsilon \mathrm{D}^{-\frac{1}{2}}\left(\Sigma \eta^{2} \Sigma \zeta^{2}-\Sigma \eta \zeta . \Sigma \eta \zeta\right)^{\frac{2}{2}} ; . . .
\end{aligned}
$$

as is found on expanding and simplifying. The probable errors in $b$ and $e$ can of course be similarly expressed.

12. In order to obtain a preliminary notion of the conditions of the problem without undue labour, I have considered a simplified system which is not strictly speaking a dynamically possible one, but which represents the actual system sufficiently nearly for the purpose in view. The observer is supposed to be carried uniformly round the sun in a circular orbit whose radius $R$ is equal to the mean radius of the earth's orlbit, the time of revolution being une year. The Jovian system is supposed to move in a very slightly elliptic orbit, with the sun at the certre of the ellipse, the inclination, $\phi$, of this orbit to that of the observer being $1^{0} 18^{\prime} 41^{\prime \prime}$, which is the actual inclination of Jupiter's orbit to the plane of the ecliptic. The excentricity of the modified orbit of Jupiter is such that its projection on the plane of the observer's orbit is a circle, this circle being unitormly described in 11 years 315 days, and having a radius $\mathrm{R}^{\prime}$ equal to the mean radius of Jupiter's actual orbit. To take account of the fact that eclipse observations are impracticable for some time before and after Jupiter is in conjunction, an eclipse is considered as possibly observable only when the angle earth-sun-Jupiter lies between the limits $\pm \psi\left(\psi=102^{\circ}\right.$, say $)$. Between these limits, eclipse observations are taken to be uniformly frequent and uniformly weighty. With the axis of $a$ through the node of Jupiter's (modified) orbit, the axis of $y$ in the perpendicular direction in the plane of the ecliptic, and the axis of $\approx$ perpendicular to the ecliptic, the results found are

$$
\left.\begin{array}{l}
\text { (p.e. in } a)^{2}=\frac{2 V^{4} \epsilon^{2}}{n} \cdot \frac{1}{\mathrm{R}^{2}-2 \mathrm{R}^{\prime} \mathrm{R} \sin \psi / \psi+\mathrm{R}^{\prime 2}} \\
\text { (p.e. in } b)^{2}=\frac{2 \mathrm{~V}^{4} \epsilon^{2}}{n} \cdot \frac{1}{\mathrm{R}^{2}\left(1-\sin ^{2} \psi / \psi^{2}\right)} \\
(\text { p.e. in } c)^{2}=\frac{2 V^{4} \epsilon^{2}}{n} \cdot \frac{\mathrm{R}^{2}-2 \mathrm{R}^{\prime} \mathrm{R} \sin \psi / \psi+\mathrm{R}^{\prime 2}}{\sin ^{2} \phi \mathrm{R}^{2} \mathrm{R}^{\prime 2}\left(1-\sin ^{2} \psi / \psi^{2}\right)}
\end{array}\right\} .
$$

13. In these expressions $R=148 \times 10^{6} \mathrm{kilom} ., \mathrm{R}^{\prime} / \mathrm{R}=\mathbf{5} \cdot \mathbf{2}$, $\mathrm{V}=3 \times 10^{3}$ kilom. per second, $\phi=1^{\circ} 18^{\prime} 41^{\prime \prime}$; $n$, the number 
of available observations, is about 330 ; and $\epsilon$, the "probable" discrepancy between the observed and calculated time of an eclipse, is estimated by Prof. Sampson at about 4.5 seconds. The numcrical values thus derived from (10) are

$$
\left.\begin{array}{rl}
\text { p.e. in } a & =44 \cdot 0 \text { kilom. per second } \\
, \quad, b & =237 \quad, \quad, \\
,, e & =10,000 "
\end{array}\right\} . .
$$

14. The determination of the velocity-component $e$ (perpendicular to the plane of the ecliptic) is so badly conditioned that the investigation can hardly be considered to afford any light on that point. It may be admitted as probable that the velocity of the solar system through the other is very far below 10,000 kilom. per second; otherwise we should have to suppose (for example) that practically all stars whose radial velocities have been measured are moving through the ather with velocities of thousands of kilometres per second. This certainly appears unlikely, though perhaps the possibility ought not to be too lightly denied. An indirect argument against a very high velocity of the solar system may be derived from other considerations. For we know that the velocity components in the plane of the ecliptic must be relatively moderate, otherwise there would be a marked anomaly, having a period of about 12 years, in the observed times of the eclipses of Jupiter's satellites*, and a priori it is unlikely that the velocity of the solar system should be nearly in some arbitrarily assigned direction, such as that perpendicular to the plane of the ecliptic.

15. On the other hand, if we begin by admitting complete ignorance regarding the velocity-components to be determined-save only our assumption that they are small compared with the velocity of light-then 10,000 kilom. per second will represent approximately the probablo error in the value of the component $c$; and in general the determination of the component of velocity resolved in any direction in space will be affected by a large probable error arising from the uncertainty in the value of $c$. On this view, the exact choice of coordinate axes becomes of importance, the relatively large probable error in $l$ given by (11) being due to the influence of the error in $c$. So long as the axes remain as specified in $\$ 12$, this unduly large error in $b$ can only be avoided (if at all) by an excessive arbitrary weighting of the observations, so designed as to secure the vanishing"

* It may be hoped that the investigation now proposed will indicate these components more definitely, or at least a superior limit to them. 
of the coefficient of $c$ in each of the first two of equations (5); these two equations being then solved for $a$ and $b$.

16. In the simplified problem of $\$ 12$, it appears that, without special weighting of the observations, the probable error in the velocity-component $b$ can be greatly reduced by a slight change of axes. The axis of $x$ remaining unchanged, the axes of $y$ and $z$ are to be rotated in their own plane through a small angle; and if the velocity-components along the new axes are called $a, b_{1}, c_{1}$, it is found that the probable error in $b_{1}$ is a minimum when the axis of $y$ lies very nearly in the plane of Jupiter's (modified) orbit. If we take that plane as the plane of $x y$, the probable errors, corresponding to our simplified problem, are:

$$
\left.\begin{array}{rlrl}
\text { p.e. in } a & =44 \cdot 0 \text { kilom. per second } \\
, ", l_{1} & =45 \cdot 5 \quad, & , \\
, " c_{1} & =10,000, & ,
\end{array}\right\} ; .
$$

using 330 observations, each with a probable error of 4.5 seconds.

17. In the absence of definite reasons to the contrary, it would be natural to take the plane of the ecliptic as one of the coordinate planes, but it appears that, by taking the plane of Jupiter's orbit instead, we can avoid increasing the probable error of one velocity-component $\left(b\right.$ or $\left.b_{1}\right)$ by an unknown amount, and this without adding seriously to the labour of computation. In the actual problem, therefore, it will probably be advisable to determine the values of two velocitycomponents in the plane of Jupiter's orbit, using for this purpose equations corresponding to the first two of (5), and causing the coefficients $\Sigma \zeta \xi, \Sigma \eta \zeta$ to disappear as nearly as may seem necessary by a moderate special weighting of the observations. We may then proceed more tentatively, on the assumption that $\mathrm{c}$ is moderate, to identify the velocitycomponent $b_{1}$ so found with the corresponding component $b$ in the plane of the ecliptic. If, for example, on general grounds, and in the absence of any positive information, we assume $c=0 \pm 500$ kilometres per second, the probable error in 6 thus arising will be approximately 12 kilom. per second, and the total probable error in $b$ will be about $\sqrt{ }\left(45 \cdot 5^{2}+12^{2}\right)$ $=47$ kilom. per second.

I hope very shortly to consider this problem more in detail; meanwhile I wish to thank Prof. Sampson for his kind and most helpful advice.

Boar's Hill, near Oxford, 9 October, 1909. 